\title{
DIE BEZIEHUNGEN POLENS ZU DEN MAGHREB-STAATEN
}

\author{
Von H. L. KRONBERger
}

Will der Betrachter des politischen Geschehens auf der internationalen Bühne die Beziehungen der Volksrepublik Polen zu Ländern der Dritten Welt untersuchen und exakt definieren, wird er zunächst von der Existenz und Kenntnis fundamentaler Prinzipien kommunistischer Außenpolitik, sodann von der aktuellen Interessenlage des polnischen Staates - freilich im Rahmen der gemeinsamen Ziele und der jeweiligen Richtlinien im sowjetischen Hegemonialbereich - auszugehen haben. Er wird demzufolge in Betracht zu halten haben, daß das polnische Verhältnis zu jenen Ländern vor allem die nachstehenden, für die gesamte Außenpolitik verbindlichen Orientierungspunkte impliziert:

1. Das Prinzip des proletarischen Internationalismus. (E. Gierek, Erster Sekretär des Zentralkomitees der PZPR ${ }^{1}$.)

2. Die Gewährleistung vorteilhafter Voraussetzungen und Bedingungen zur Errichtung sozialistischer und kommunistischer Verhältnisse in der Welt. (A. Gromyko, Außenminister der UdSSR.)

3. Die Verstärkung von Einheit und Geschlossenheit der Staaten in der sozialistisch-kommunistischen Gemeinschaft und die Festigung der Freundschaft und des brüderlichen Verhältnisses unter ihnen. (A. Gromyko.)

4. Die Unterstützung der nationalen Befreiungsbewegungen und die umfassende Zusammenarbeit mit den jungen Entwicklungsländern. (A. Gromyko.)

5. Die konsequente Verteidigung der Grundsätze der friedlichen Koexistenz von Staaten unterschiedlicher Gesellschaftssysteme. (A. Gromyko.)

6. Die Maxime, daß die Politik der friedlichen Koexistenz nicht die Verewigung gemeinsamen Daseins, des Zusammenlebens, zugrundelegt, vielmehr den Rahmen für Klassenauseinandersetzungen zwischen den entgegengesetzten gesellschaftlichen und politischen Systemen schafft. Damit wird zugleich die Frage des ideologischen Kampfes in die vordere Reihe gerückt.

Der unerschütterliche Grundsatz des proletarischen Internationalismus in der Außenpolitik schließt nun freilich die Wahrnehmung eigener polnischer Interessen keineswegs aus. Er bildet ihren ideologischen Überbau. Folgerichtig hatte denn auch der VI. Parteitag der PZPR im Dezember 1971, im Zusammenhange mit der Bestimmung der Grundrichtungen der Außenpolitik des Landes, als die wichtigsten Zukunftsaufgaben bezeichnet:

1. Die Vertiefung des Bündnisses und der Zusammenarbeit mit der Sowjetunion und den anderen kommunistischen Staaten.

2. Die Festigung der kommunistischen Gemeinschaft und deren Stellung in der Welt.

3. Die Konsolidierung von Sicherheit und Frieden (im kommunistischen Verständnis).

4. Die Gewährleistung vorteilhafter Voraussetzungen und Bedingungen einer günstigen Realisierung des gesellschaftlich-wirtschaftlichen Entwicklungsprogramms für den kommunistischen polnischen Staat.

1 PZPR = Abkürzung für Polska Zjednoczona Partia Robotnicza, zu deutsch: Polnische Vereinigte Arbeiterpartei (PVAP). 
Diese Aufgaben wurden als eine Einheit begriffen, die aus dem Klassencharakter kommunistischer Außenpolitik ihre Impulse erhält.

Es ist in hohem Maße bezeichnend für das starke Selbstbewußtsein der polnischen Parteiführung, wenn sie für sich das Verdienst in Anspruch genommen hat, die besonderen, charakteristischen Merkmale der weltpolitischen Epoche vor dem VI. Parteitag erkannt und in adäquater Weise - somit auch für die Gesamtinteressen Polens - genutzt zu haben. Unter Hinweis auf die bedeutendsten Entwicklungstendenzen in der zeitgeschichtlichen, internationalen Lage, hatte der Parteitag - gemäß den Weisungen für den gesamten Ostblock - die Führung der Partei und die Regierung gleichermaßen zu einer aktiven Außenpolitik verpflichtet. In den Erfolgen der solchermaßen geführten Außenpolitik der Staaten in der sozialistisch-kommunistischen Gemeinschaft bei der internationalistischen Unterstützung der Außen- und Innenpolitik der Entwicklungsländer sieht die polnische Außenpolitik auch ihren eigenen, besonderen Anteil, als Ergebnis spezifischer Maßnahmen auf internationaler Ebene ${ }^{2}$. Die Bestätigung dieser bemerkenswerten Selbsteinschätzung kam in dem zum VII. Parteitag ${ }^{3}$ der PZPR erstatteten Rechenschaftsbericht des Zentralkomitees zum Ausdruck. Darin wurde festgestellt:

In der Berichtszeit zwischen dem VI. und dem VII. Parteitag machten Verstärkung und Vertiefung der politischen, wirtschaftlichen und kulturellen $\mathrm{Zu}$ sammenarbeit Polens mit den Entwicklungsländern weitere Fortschritte ${ }^{4}$.

Auch die hohe Aktivität der Partei in bestimmten Entwicklungsländern auf außenpolitischem und gesellschaftspolitischem Gebiet vor allem fand in dem Bericht ihre Anerkennung:

Die Zusammenarbeit der Parteien wie auch die von der PZPR stimulierten Kontakte polnischer gesellschaftlicher Organisationen $\mathrm{zu}$ den entsprechenden Organisationen in Entwicklungsländern haben auch ein besseres politisches Klima zur Erweiterung der Zusammenarbeit zwischen Polen und vielen Entwicklungsländern geschaffen ${ }^{5}$.

Wie die beiden führenden Politiker in Polen, der Erste Sekretär des Zentralkomitees der PZPR, E. Gierek, und der Vorsitzende des Ministerrats, P. Jaroszewicz, auf dem VII. Parteitag angekündigt haben, soll die bisherige, erfolgreiche Politik enger Zusammenarbeit polnischer staatlicher Institutionen, des Zentralkomitees der polnischen Partei und polnischer gesellschaftlicher Organisationen mit den entsprechenden Einrichtungen in vielen Entwicklungsländern fortgeführt, ja, noch bedeutend erweitert und vertieft werden. Das gilt für die wirtschaftlichen Beziehungen, die Kooperation im industriellen Bereich und die wissenschaftlich-technische Zusammenarbeit. Das gilt aber nicht minder für das Zusammenwirken der kommunistischen Parteien insgesamt, somit auch der polnischen sozialistischen kommunistischen Partei, mit den sogenannten fortschrittlichen Bewegungen und mit den z. T. revolutionären Zusammenschlüssen für nationale Befreiung ${ }^{6}$.

Siehe Longin Zareba in Gazeta Lubuska, Nr. 274 vom 10. 12. 1975.

$3 \mathrm{Er}$ wurde in Warschau vom 8. bis 12. 12. 1975 abgehalten.

4 Siehe Glos Szczecinski, Nr. 271 vom 6. 12. und 7. 12. 1975.

5 Siehe ebenda.

6 Siehe Glos Wybrzeza, Nr. 273 vom 9. 12. 1975 und Glos Szczecinski, Nr. 274 vom 10.12 .1975$. In der Zusammenarbeit ist der polnische Gewerkschaftsverband auch sehr aktiv. 


\section{Marokko}

Als die mit dem Ausbruch des Zweiten Weltkrieges jäh abgebrochenen wirtschaftlichen Beziehungen zwischen Polen und Marokko bald nach Beendigung der Kriegshandlungen wiederaufgenommen wurden, konnten die Partner auf eine fast zwei Jahrzehnte lange Tradition zurückblicken. Die Partei- und Staatsführung des kommunistischen Polens ging dabei von ökonomischen Überlegungen, im Interesse der eigenen Volkswirtschaft, aus. Ihre Argumentation gründet sich vor allem auf Erfahrungen mit dem Bau kompletter Industriebetriebe verschiedener Art in den Entwicklungsländern durch polnische Unternehmen ${ }^{7}$. Die bedeutendsten Auftraggeber für polnische industrielle Investitionen im Mittelmeerraum sind die arabischen Länder, unter denen Marokko - neben dem Irak - an der Spitze steht. $\mathrm{Zu}$ den größten und wichtigsten Investitionen in diesem Lande zählt der im Jahre 1974 in Angriff genommene Bau einer Schwefelsäurefabrik mit einer Tageskapazität von eintausend Tonnen ${ }^{8}$. Zuvor hatte die für Investitionen im Auslande zuständige staatliche Außenhandelsfirma in Warschau den Auftrag zur Beteiligung am Bau eines Kombinats für Mineraldünger in Marokko erhalten. Im einzelnen hatte der polnische Partner im Rahmen der Beteiligung zwei komplette Schwefelsäurefabriken zu liefern ${ }^{9}$. Dieser Auftrag wurde später in dem Sinne erweitert, daß eine dritte Fabrik für Schwefelsäure gebaut werden sollte, um die benötigten Mengen dieses chemischen Produkts für die im Aufbau befindliche Fabrik für Phosphorsäure in Safi zu gewährleisten ${ }^{10}$. Das Vorhaben in Safi wird übrigens von einem Konsortium von Firmen aus der Bundesrepublik und aus Polen unter der Firmenbezeichnung "Uhde - Siemens - Polimex" ausgeführt, während für die Schwefelsäurefabriken der Rohschwefel aus Polen eingeführt wird ${ }^{11}$. Schließlich bekundete der polnische Außenhandel hohes Interesse an den marokkanischen Plänen, die Phosphatvorkommen im verstärkten Ausmaß abzubauen und im eigenen Lande $\mathrm{zu}$ verarbeiten. $\mathrm{Zu}$ dem Zweck sollen sieben Kombinate für die Erzeugung von Phosphorsäure bis 1980 gebaut werden ${ }^{12}$. Im Juni 1974 wurden sodann von den beiden Partnern ein Handelsabkommen für mehrere Jahre und ein Protokoll zum Übergang von der bis dahin praktizierten Verrechnung im Clearingverfahren auf die Verrechnung im freien Devisenverkehr unterzeichnet.

Das Abkommen sah einen dreifachen Anstieg der Warenumsätze allein im Jahre der Unterzeichnung, im Vergleich mit denen im Jahre 1973, vor; er wurde auf rund 360 Millionen Devisen-Zloty ${ }^{13}$ beziffert. In dem Handelsvertrag steht dem marokkanischen Export vor allem von Phosphat, Phosphorsäure, Fischmehl, Eisenerz, Buntmetallerz, landwirtschaftlichen Produkten, Lebensmitteln und Industrieerzeugnissen die polnische Ausfuhr von Schwefel, Zucker, Steinkohle, Erzeugnissen der chemischen Industrie und des Maschinenbaus sowie von verschiedenen Investi-

7 Siehe J. Pacula in Express Wieczorny, Nr. 90 vom 16. 4. 1974.

8 Siehe ebenda.

9 Siehe Trybuna Ludu, Nr. 234 vom 23. 8. 1973.

10 In der Förderung von Phosphat steht Marokko in der Spitzengruppe der Länder mit solchen Vorkommen. Siehe Z. Skowron in Trybuna Ludu, Nr. 130 vom 10. 5. 1974.

11 Zur Lieferung polnischen Schwefels an Marokko und andererseits von marokkanischem Phosphat an Polen wurde Ende Juli 1973 in Rabat ein Abkommen unterzeichnet. Siehe Trubuna Ludu, Nr. 213 vom 2. 8. 1973. Das Vorhaben steht unter der Schirmherrschaft der Weltbank. Siehe Trybuna Ludu, $\mathrm{Nr} .184$ vom 3. 7. 1974.

12 Siehe Z. Skowron in Trybuna Ludu, Nr. 130 vom 10. 5. 1974.

13 Ein Devisen-Zloty ist die Verrechnungseinheit im Außenhandel. Er entspricht einem Verrechnungswert von 0,25 US-Dollar bzw. 0,225 Rubel. 
tionsgütern gegenüber. Schon zu dem Zeitpunkt wurde Marokko als einer der bedeutendsten Handelspartner Polens unter den Entwicklungsländern bezeichnet. Darüber hinaus wurde in Warschau die günstige Entwicklung der Wirtschaftsbeziehungen sowohl im Warenaustausch wie auch unter dem Aspekt einer recht weit begriffenen Zusammenarbeit im wirtschaftlichen Bereich hervorgehoben ${ }^{14}$.

Die forcierte, hohe Spezialisierung der polnischen Investitionsgüterindustrie ${ }^{15}$ vor allem hat den Ausschlag gegeben, wenn die erste Zuckerfabrik in Marokko (in El Belar) von Fachleuten aus Polen erbaut wurde. Dabei kam dem wirtschaftlichen Interesse Warschaus an den gesamten Entwicklungsvorhaben der marokkanischen Regierung deren Beschluß entgegen, den Zuckerbedarf des Landes sukzessive aus Eigenerzeugung zu decken.

Konsequentes Vorgehen des polnischen Außenhandels und konzentrierte Bemühungen - übrigens in mehreren ausgewählten Ländern - führten auch in einem anderen Bereich der Wirtschaftsbeziehungen zu Marokko zu konkreten und positiv gewerteten Abschlüssen. Mit den im April 1974 unterzeichneten drei Lieferverträgen für Elektro- und Diesellokomotiven, Personenwagen und Zysternenwaggons zum Transport von Phosphorsäure konnte die Position der einschlägigen polnischen Industrie auf dem marokkanischen Markt, als wichtigster Lieferant für rollendes Eisenbahnmaterial gefestigt werden ${ }^{16}$.

In die Zukunft gerichtete Überlegungen auf polnischer Seite und durch die ersten Lieferungen erlangtes Renommee haben sich somit, nicht zuletzt noch gefördert durch die lange Tradition ${ }^{17}$, als sehr nützlich für die Industrie und den Außenhandel Polens erwiesen. Im Ergebnis konnte Polen im Außenhandel und in der wirtschaftlichen Zusammenarbeit des nordafrikanischen Königreichs mit den Ländern im Ostblock Anfang 1975 den ersten Platz einnehmen.

Auch personell ist der polnische Partner in Marokko präsent - in Gestalt vor allem von Gruppen aus Technikern, Facharbeitern, Spezialisten und Instrukteuren ${ }^{18}$.

Demgegenüber ist die polnische Präsenz auf andern Gebieten vergleichsweise bescheiden. Sie beschränkte sich im wesentlichen auf wissenschaftlich-technische Kontakte mit einschlägigen marokkanischen Institutionen, auf eine Zusammenarbeit in den Bereichen der Kultur, der Kunst und des Sports ${ }^{19}$, auf Inventarisierungsarbeiten polnischer Studenten für Baudenkmäler in der ehemaligen Hauptstadt Marokkos, Medina de Fez, unter der Leitung der UNESCO ${ }^{20}$, und auf die

14 Die vereinbarte Lieferung von $\mathrm{rd}$. 400000 Tonnen Phosphorsäure und großer Mengen Phosphat an Polen in den Jahren 1974 bis 1980 ist für die polnische Industrie von großer Bedeutung.

15 Polen hat eine Art Monopolstellung im Rahmen der internationalen Arbeitsteilung unter den Mitgliedsländern des Rats für Gegenseitige Wirtschaftshilfe, was die schlüsselfertige Lieferung kompletter Schwefelsäurefabriken und Zuckerfabriken betrifft. Siehe J. Pacula in Express Wieczorny, Nr 90 vom 16. 4. 1974.

16 Marokko ist nach der Sowjetunion der zweitgrößte Absatzmarkt der Eisenbahnindustrie Polens. Zwei Drittel der für den Ankauf von Lokomotiven und Waggons veranschlagten Beträge in seinem Budget stellte das Land für Lieferungen aus Polen zur Verfügung. Siehe H. Chadzynski in Zycie Warszawy, Nr. 92 vom 18. 4. 1974.

17 Eine der ersten Dampflokomotiven der marokkanischen Eisenbahn wurde in den zwanziger Jahren dieses Jahrhunderts von Polen geliefert. In den Jahren 1967 bis 1970 erfolgten, mit insgesamt 420 Güterwaggons, die ersten Nachkriegslieferungen aus Polen. Die Waggons wurden überwiegend in den Güterwaggons, die ersten Nachkriegslieferungen aus Polen. Die Waggons wurden überwiegend in den 92 vom 18. 4. 1974, Trybuna Ludu, Nr. 2 vom 3.1. 1975 und PAP in Trybuna Odrzanska, Nr. 205 vom 18. 9. 1975.

18 Z. B. auf dem Güterbahnhof des Hafens in Casablanca; siehe H. Chadzynski in Zycie Warszawy, Nr.

19 Eine entsprechende Vereinbarung wurde in Rabat im Oktober 1969 unterzeichnet. Siehe Zycie Warszawy, Nr. 261 vom 1. 11. 1969.

20 Siehe Trybuna Ludu, Nr. 224 vom 12. 8. 1974. 
Polnischen Kulturtage in Rabat, Ende Oktober 1974, im Rahmen von Veranstaltungen aus Anlaß des dreißigjährigen Bestehens der Volksrepublik Polen ${ }^{21}$.

Noch bescheidener nimmt sich die erkennbare politische Aktivität aus. Nichtsdestoweniger zielt ihre Richtung unverkennbar auf die Veränderung der innenpolitischen Struktur und des Gesellschaftssystems im Königreich und auf die zumindest politische, ideologische und propagandistische - Unterstützung der linken Kräfte, des sogenannten Fortschritts und des Sozialismus. Das Zentralkomitee der Polnischen Vereinigten Arbeiterpartei nahm die Gründung der Partei des Fortschritts und des Sozialismus in Marokko im Spätsommer 1974 zum Anlaß, in einem Telegramm an das Zentralkomitee dieser Partei die größten Erfolge in der Verwirklichung ihrer programmatischen Ziele und im weiteren Kampf der Arbeitermassen in Marokko um gesellschaftspolitischen Fortschritt, Demokratie des Volkes und soziale und gesellschaftliche Gerechtigkeit zu wünschen. Die polnische Parteiführung brachte außerdem ihre brüderliche, internationalistische Solidarität mit dem innenpolitischen Kampf der Arbeitermassen, unter der Führung jener kommunistischen Partei, im Königreich zum Ausdruck ${ }^{22}$.

Dieser Vorgang war nicht ohne eine gewisse außenpolitische Pikanterie, nachdem nur etwa sieben Wochen vorher ein Sonderbeauftragter König Hassans II., in Begleitung des marokkanischen Botschafters in Rumänien, in der polnischen Hauptstadt sich aufgehalten hatte, um dem Vorsitzenden des Staatsrats eine Botschaft seines Königs zu überbringen und anschließend im Außenministerium ein Sondierungsgespräch mit dem stellvertretenden Ressortchef Romuald Spasowski zu führen. Dabei trug der Emissär aus Rabat den Standpunkt der Regierung Marokkos zur Frage der spanischen Sahara vor ${ }^{23}$.

\section{Algerien}

Die wirtschaftlichen Beziehungen Polens zum größten Lande in Maghreb, Algerien, können - im Unterschied zu den polnisch-marokkanischen Beziehungen, indes als ganz normale Folge des früheren Status Algeriens, als eines sogenannten französischen Nebenlandes ${ }^{24}$ - nicht auf eine längere Tradition zurückblicken. Erst mit der Erlangung der Eigenstaatlichkeit im Jahre 1962 wurde die Voraussetzung zu ihrer Aufnahme geschaffen. Nach ihrer Institutionalisierung entwickelten sie sich in recht bescheidenem Umfange: Im Jahre 1968 konnte die Außenhandelsbilanz auf polnischer Seite einen Umsatz von nicht mehr als 3,3 Millionen Dollar ausweisen. Wenn auch die weitere Entwicklung eine kontinuierliche Anstiegstendenz erkennen läßt, so war der Umsatz im Jahre 1973 (rund acht Millionen Dollar) doch weiterhin eher unbedeutend ${ }^{25}$.

Als entscheidenden Durchbruch sind die Verhandlungsergebnisse einer polnischen Regierungsdelegation unter der Leitung des stellvertretenden Vorsitzenden des polnischen Ministerrats, Kazimierz Olszewski, in Algerien im August 1973 gewertet worden ${ }^{26}$. Im einzelnen wurde vereinbart:

\footnotetext{
21 Siehe Trybuna Ludu, Nr. 333 vom 29. 11. 1974.

22 Siehe Trybuna Ludu, Nr. 276 vom 3. 10. 1974.

23 Siehe Trybuna Ludu, Nr. 223 vom 11. 8. 1974

24 Algerien gehörte nicht zum französischen Kolonialbesitz und unterstand dem Innenministerium in Paris.

25 Siehe PAP in Trybuna Ludu, Nr. 160 vom 9. 6. 1974

26 Siehe ebenda.
} 
Polen gewährt Algerien einen bedeutenden - nicht bezifferten - Investitionskredit zur Ausführung von Entwicklungsvorhaben im Lande. Der Warenaustausch zwischen beiden Ländern im Jahre 1974 soll, im Vergleich mit 1972, um das Vierfache zunehmen ${ }^{27}$. Es wird ein Polnisch-Algerisches, Ständiges Komitee beider Regierungen ins Leben gerufen, das die weitreichende $\mathrm{Zu}-$ sammenarbeit und den Erfahrungsaustausch perspektivisch planen und koordinieren soll28. $\mathrm{Zu}$ einem bestimmten Zeitpunkt werden beide Seiten mit Verhandlungen zum Abschluß eines langfristigen Handelsvertrags ${ }^{29}$ beginnen ${ }^{30}$.

In den Gesprächen wurde darüber hinaus der künftige Umfang des bilateralen Warenaustauschs festgelegt und schließlich über die Möglichkeiten der Lieferung kompletter Industriebetriebe und von Investitionsgütern aus Polen diskutiert ${ }^{31}$.

In den Verhandlungen sind nicht alle Erwartungen Warschaus erfüllt worden, nachdem der Leiter der polnischen Delegation nach deren Rückkehr in einer Erklärung die Festlegung eines langjährigen Programms der wirtschaftlichen Zusammenarbeit als das hauptsächliche Ziel der Reise nach Algerien genannt hatte ${ }^{32}$. Diese Zielsetzung ist vor dem Hintergrunde der bedeutenden Rohöl- und Erdgasvorkommen Algeriens zu sehen. Freilich wäre das gleichwohl auch für den polnischen Partner positive Ergebnis der Verhandlungen ohne das auf algerischer Seite bekundete - auch von Staatspräsident Boumedienne erklärte - Interesse an einer Zusammenarbeit mit den kommunistischen Ländern insgesamt und mit Polen im anstehenden Falle nicht zustande gekommen ${ }^{33}$.

Einen neuen Akzent erhielten die Beziehungen zwischen beiden Ländern mit der Reise einer Abordnung des Zentralkomitees der polnischen führenden Partei (PZPR) nach Algerien im November 1973. Der Besuch erhielt schon dadurch hohes Gewicht, daß an der Spitze der Funtionäre aus Warschau das Mitglied des Politbüros und der Sekretär des Zentralkomitees der Partei, Edward Babiuch, stand, der in der Parteihierarchie als sehr enger Vertrauter Edward Giereks gilt. Während die Gastgeber den Besuch als neuen, bedeutsamen Schritt auf dem Wege der Entwicklung direkter Zusammenarbeit der polnischen kommunistischen Partei mit der führenden politischen Kraft im algerischen Staat ${ }^{34}$ interpretierten, hob der Repräsentant der polnischen Abordnung in seiner ersten Erklärung die Solidarität der polnischen Nation mit dem algerischen Volk und mit dessen Kampf um die Unabhängigkeit in letzter Vergangenheit hervor. Zugleich bekundete er den Wunsch seiner Parteiführung nach engerer Zusammenarbeit mit der Befreiungsfront, aber auch nach gemeinsamem Kampf gegen Imperialismus und Neokolonialismus ${ }^{35}$.

\footnotetext{
27 Die Partner gingen dabei sogar von der Erwartung aus, daß durch die Schaffung zusätzlicher Möglichkeiten der Aktivierung der Austausch im Jahre 1974, im Vergleich mit 1973, einen zehnfachen Anstieg (rund 80 Millionen Dollar) erzielen werde. Siehe PAP vom 8. 6. 1974.

$28 \mathrm{~K}$. Olszewski nach Rückkehr der Delegation in Warschau. Siehe PAP vom 22. 8. 1973.

$29 \mathrm{Er}$ soll sich auf mindestens zehn Jahre erstrecken.

30 Für Polen standen Lieferungen algerischen Rohöls und algerischer Erzeugnisse aus Rohöl an vorderer Stelle.

31 Siehe 28.

33 Siehe PAP vom 22, 8, 1973 und vom 9, 6, 1974.

34 Gemeint ist die Front der Nationalen Befreiung.

35 Siehe Ignacy Krasicki in Trybuna Ludu, Nr. 313 vom 10. 11. 1973.
} 


\section{Ideologie, Gesellschaftspolitik und Außenpolitik als Stimulans wirtschaftlicher Zusammenarbeit}

Wenn auch die Erörterung beide Länder interessierender Fragen der Wirtschaft und des Außenhandels in angemessenem Umfange stattfand ${ }^{36}$; ist das wichtigste Ergebnis des Besuchs der polnischen Parteidelegation doch auf der ideologischen, gesellschaftspolitischen und außenpolitischen Ebene festzustellen. Mit anderen Worten: In den Beziehungen zwischen den beiden Ländern wurde mit der Einführung einer politischen Komponente der bis zu diesem Zeitpunkt überwiegend wirtschaftlichen und kulturellen Zusammenarbeit die künftige, sehr enge, dynamisch geplante Kooperation auf den genannten Ebenen hinzugefügt. Mit seiner Schilderung des Aufbaus sozialistischer Verhältnisse - vor allem der Entwicklung der Wirtschaft nach $1970^{37}$ in Polen verfolgte der Leiter der polnischen Parteifunktionäre den Zweck, werbende Wirkung im Hinblick auf die Entwicklung zu kommunistischen Gesellschaftsstrukturen in Algerien zu erzielen ${ }^{38}$. Nach den Worten des algerischen Handelsministers Jaker sollte der polnische Investitionskredit beitragen, die Pläne der algerischen Führung zur Veränderung der Wirtschaftsstruktur nach sozialistischem Muster zu verwirklichen ${ }^{39}$. Die Erfahrungen der polnischen kommunistischen Partei wurden auf algerischer Seite als nützlich und wertvoll für die gesellschaftspolitischen Veränderungen und Reformen ${ }^{40}$ im eigenen Lande gewertet ${ }^{41}$. Der Regierungsebene in den bilateralen Beziehungen beider Länder hat sich die politisch-ideologische Ebene zugesellt ${ }^{42}$. Das polnische Politbüro-Mitglied äußerte seine Überzeugung von einem raschen Fortschritt Algeriens auf dem Wege zur sozialistischen Entwicklung in Wirtschaft, Gesellschaft und Kultur. Er sagte die Unterstützung dieser Entwicklung durch die PZPR zü3. In der Beurteilung der internationalen Lage bestätigten beide Seiten ihre Solidarität. Beide Seiten vereinbarten enge Zusammenarbeit und Erfahrungsaustausch zwischen ihren politischen Parteien in allen Bereichen der Politik ${ }^{44}$. Die Parteiführung Polens verfolgt aufmerksam die außenpolitische, gesellschaftspolitische und soziale Entwicklung in den arabischen Ländern und in der Dritten Welt. Vermittels verstärkter, engerer Ver-

\footnotetext{
36 Gesprächs- und Verhandlungspartner waren der Vorsitzende des Revolutionsrats und der Regierung, Staatspräsident Huari Boumedienne, das Mitglied des Revolutionsrats und der Staatsminister Sheriff Belkasem, der Minister für Industrie und Energiewesen, Abt Es-Salem Belaid, der Minister für Hochschulwesen und Wissenschaft und Vorsitzende der Landeskommission für Jugendfragen, Mohammed Seddik-Ben Jahia, der Handelsminister Lajasz Jaker u. a. m. Auf beiden Seiten wurde das komplemenSëdik-Ben Jahia, der Handelsminister Lajasz Jaker u. a. m. Auf beiden Seiten wurde das komplemen-
täre Symptom der Wirtschaftssysteme beider Länder, vor allem bezüglich des Rohstoffbedarfs, hervorgehoben. Siehe PAP vom 10.11. 1973.

37 Nach den z. T. blutigen Arbeiterunruhen in den polnischen Hafenstädten Gdansk (Danzig) und Gdynia (Gdingen) im Dezember 1970, als Folge weitverbreiteter Unzufriedenheit und Empörung über Preiserhöhungen für manche Grundnahrungsmittel - bei ausbleibenden Lohnerhöhungen und gleichzeitigem Mangel an den wichtigsten Gütern des täglichen Bedarfs - wurde das gesamte GomulkaRegime abgesetzt und Edward Gierek mit der politischen Führung, durch seine Wahl zum ersten Sekretär des ZK der PZPR, beauftragt. Seine Wirtschafts- und Sozialpolitik ist durch hohe Dynamik gekennzeichnet.

38 Siehe Ignacy Krasicki in Trybuna Ludu, Nr. 314 vom 11. 11. 1973.

39 Jaker sprach von einer grundlegenden Veränderung der Struktur der gesamten Wirtschaft. Algerien befinde sich in einer Zeit des Umbruchs. Siehe PAP vom 10. 11. 1973.

$40 \mathrm{Im}$ Rahmen der Agrarrevolution und der Einführung sozialistischer Verhältnisse in den vergesellschafteten Produktionsbetrieben. Siehe J. Krasicki in Trybuna Ludu, Nr. 316 vom 13. 11. 1973.

41 Zitat eines Funktionärs der algerischen Befreiungsfront: "Sie sind eine zuverlässige Stütze und Quelle wertvollen gesellschaftspolitischen Wissens für die Front der"Nationalen Befreiung “. Siehe Quelle 51.

42 Wörtlich: "Eine wesentliche Prämisse der weiteren Annäherung des antiimperialistischen und sich fortschrittlich entwickelnden Algeriens an Polen, jenes aktive und wichtige Glied der Gemeinschaft der sozialistischen Staaten.“ Aus Außerungen algerischer Funktionäre in Betriebsversammlungen. Siehe J. Krasicki in Trybuna Ludu, Nr. 314 vom 11. 11. 1973.

43 Siehe PAP vom 12. 11. 1973.

44 Aus dem gemeinsamen Kommuniqué vom 12.11. 1973. Quelle siehe 56.
} 
bindung und Zusammenarbeit mit der Nationalen Befreiungsfront in Algerien wünscht die polnische Parteiführung, Verbindung auch $z u$ allen sozialistischen und antiimperialistischen Kräften in der Welt zu knüpfen ${ }^{45}$.

Die Reise der polnischen Parteidelegation war ganz bewußt, im Blick auf die aktuellen Ereignisse vor allem im Vorderen Orient, aber auch im Hinblick auf die gesellschaftspolitischen Veränderungen in Algerien ${ }^{46}$, und zielstrebig in einer als besonders günstig erscheinenden Zeit ${ }^{47}$ geplant und ausgeführt worden. Die Intentionen der Führung in Warschau liefen darauf hinaus, das besondere Interesse der politischen Kreise, aber auch der Bevölkerung Algeriens am weiteren Ausbau der Beziehungen vor allem zu Polen, darüber hinaus indessen auch zum gesamten Ostblock, zu wecken ${ }^{48}$. Man wird somit, nach den Erfahrungen und Kenntnissen in bezug auf zwischenstaatliche, außenpolitische Initiativen und Aktionen von Staaten im Machtbereich der Sowjetunion, von der These ausgehen dürfen, daß der Besuch zumindest in der sogenannten sozialistischen Gemeinschaft koordiniert, wenn nicht sogar von der sowjetischen Führung initiiert war. Für die Wahl und die Aufgabe gerade der polnischen Parteiführung im algerischen Engagement ist die Prämisse entscheidend gewesen, daß die Außenpolitik Polens als besonders aktiv und dynamisch gilt ${ }^{49}$. In dem Wunsche, alle gegebenen und fürderhin sich bietenden Möglichkeiten zum Ausbau der bilateralen Beziehungen zu nutzen, verfolgt die Führung in Warschau das Ziel - es ist sowohl aktuell als auch perspektivisch angelegt - , mit Algerien in den Bereichen der Innenpolitik (freilich beschränkt auf den algerischen Raum) und der Außenpolitik auf das engste zusammenzuarbeiten. Aus der übergeordneten Tatsache der politischen Gemeinschaft sollen praktische Folgerungen gezogen werden ${ }^{50}$.

Was nun die Folgerungen, d. h. die praktische Anwendung der vereinbarten Grundsätze zur Politik der Freundschaft und Zusammenarbeit, somit deren Handhabung, im wirtschaftlichen Bereich anlangt, wurde in der ersten Sitzung des Polnisch-Algerischen, Gemischten Komitees im Juni 1974 in Warschau ein bedeutender Fortschritt in der Entwicklung des Warenaustausches zwischen beiden Ländern festgestellt ${ }^{51}$. Nach einem erzielten Volumen in Höhe von 25 Millionen Dollar im ersten Quartal 197452 wurde mit einem Gesamtumsatz von rund 80 Millionen Dollar im Jahre 1974 gerechnet. Die größten Entwicklungsmöglichkeiten für die Zusammenarbeit erblickten die Partner im Schiffbau, in der chemischen und petrochemischen Industrie, in der Baustoffindustrie, im Maschinenbau, in der Holz- und in der Nahrungsmittelindustrie ${ }^{53}$.

Eine weitere Form polnischer Präsentation ${ }^{54}$ in Algerien war die in der Hauptstadt dieses Landes veranstaltete Messe in der ersten Septemberhälfte 1974, insonderheit der sogenannte Polnische Tag in ihrem Rahmen. Vor allem dem Angebot von Dienstleistungen, z. B. in der gesamten Wasserwirtschaft, in der Suche nach

\footnotetext{
45 Siehe ebenda.

46 Der Warschauer Korrespondent J. Krasicki berichtete von „bedeutenden sozialistischen Umwandlungen und Reformen im Zuge der Agrarrevolution und der sozialistischen Organisation der Produktion in der vergesellschafteten Industrie “. Siehe Trybuna Ludu, Nr. 320 vom 17. 11. 1973.

47 Krasicki charakterisierte sie als „ereignisträchtig“.

48 Siehe J. Krasicki in Trybuna Ludu, Nr. 320 vom 17. 11. 1973.

49 Siehe ebenda.

50 Siehe ebenda.

51 Siehe PAP in Trybuna Ludu, Nr. 166 vom 15.6.1974: Ein bislang „nicht verzeichneter“ Fortschritt.

52 Siehe T. Jackowski in Zycie Warszawy, Nr. 221 vom 15. 9. 1974.

53 Siehe 51.

54 Aus Polen hatten 13 Außenhandelszentralen ausgestellt. Siehe 52.
} 
Naturschätzen und in der Erstellung von Expertisen zu Rohstoffvorkommen für die Baustoffindustrie, wurde hohe Bedeutung beigemessen ${ }^{55}$.

Darüber hinaus bleibt die recht bedeutende Rolle polnischer Unternehmen und Institutionen beim Ausbau des Hafens in Oran, weiter in der Inbetriebnahme des Meeresinstituts, in der Erstellung eines Städteplans für Oran und schließlich in der beruflichen Ausbildung algerischer Facharbeiter und Spezialisten ${ }^{56} \mathrm{zu}$ nennen ${ }^{57}$. Die polnische Werftindustrie hatte für die schulische und fachliche Ausbildung nach der Errichtung der Werft in Oran benötigter Arbeitskräfte an Ort und Stelle eine kleine Werft für Ausbildungszwecke entworfen, in der die Arbeit, unter der Anleitung und Aufsicht von 30 polnischen Fachkräften ${ }^{58}$, bereits aufgenommen worden ist. Den Kern der künftigen Belegschaft der Großwerft sollten 200 Algerier bilden, von denen 100 in der örtlichen Schulungswerft, die gleiche Zahl aber auf polnischen Werften ausgebildet wurden bzw. werden ${ }^{69}$. Übrigens wird die von polnischen Fachleuten entworfene Großwerft die erste algerische Werft überhaupt sein ${ }^{60}$.

Nachdem auf kulturellem und auf wissenschaftlichem Gebiet bereits im Jahre 1972 ein Abkommen zur Zusammenarbeit für das Jahr 1973 unterzeichnet worden war ${ }^{61}$, sind im April 1974 zwei Programme vereinbart worden: Ein solches zur kulturellen und wissenschaftlichen Zusammenarbeit in den Jahren 1974 und 1975 und ein weiteres zur wissenschaftlich-technischen Zusammenarbeit im Jahre 1974. Sie beinhalten vor allem die Zusammenarbeit in der Ausbildung von Wissenschaftlern und Künstlern und fixieren das Ausmaß der Beteiligung polnischer Fachkräfte an der Realisierung der algerischen Entwicklungspläne ${ }^{62}$.

Schon vor mehr als zehn Jahren hat die polnische Regierung, im Rahmen der Zusammenarbeit im Gesundheitswesen, begonnen, Arzte nach Algerien zu entsenden, wo sie in fast allen Städten tätig gewesen sind oder noch arbeiten; so z. B. in den medizinischen Einrichtungen der Universitäten in Oran und in Constantine ${ }^{63}$, außerdem in den Institutionen des Gesundheitswesens in Satiff, Biskra, Batni, ja, selbst in der Oase El-Ued. Im September 1974 war vereinbart worden, die Zusammenarbeit - sie hatte sich bereits kontinuierlich entwickelt ${ }^{64}$ - noch $\mathrm{zu}$ erweitern. Insonderheit sollten spezielle wissenschaftliche Verbindungen zwischen den Universitätskliniken und medizinischen Instituten geknüpft werden.

Auch zum Fragenkomplex der Informationspolitik und der Tätigkeit der Massenmedien ging die Initiative zur Zusammenarbeit von Warschau aus. Gesprächen des zuständigen Abteilungsleiters im algerischen Ministerium für Kultur und Informa-

55 Polnische Spezialflugzeuge für Zwecke der Land- und Forstwirtschaft sind auch in Algerien zum Pflanzenschutz in der Bekämpfung von Schädlingen auf riesigen landwirtschaftlich genutzten Flächen eingesetzt worden. Siehe Glos Wybrzeza, Nr. 3 vom 4. 1. und 5. 1. 1975.

56 Zahlreiche algerische Studenten erhielten ihre Fachausbildung in Hochschulen im Raum Danzig Zoppot - Gdingen. Mehrere Dutzend von ihnen absolvierten ihr Praktikum in der Werft in Gdingen. Siehe Zycie Warszawy, Nr. 74 vom 28. 3. 1974.

57 Siehe Trybuna Ludu, Nr. 62 vom 3. 3. 1974.

58 Die Gesamtzahl der in Algerien eingesetzten polnischen Fachkräfte der verschiedenen Berufssparten wurde Mitte 1974 auf rund 200 beziffert. Siehe PAP in Trybuna Ludu, Nr. 160 vom 9. 6. 1974.

59 Siehe PAP in Zycie Warszawy, Nr. 269 vom 12. 11. 1974.

60 Siehe T. Jackowski in Zycie Warszawy, Nr. 280 vom 24. 11. und 25. 11. 1974.

60 Ss sah u. a. vor: Die enge Zusammenarbeit von Hochschulen beider Länder in den exakten, humanistischen und landwirtschaftlichen Wissenschaften, die Zusammenarbeit des Rundfunks, des Fernsehens und der. Fotoagenturen in beiden Ländern, sodann Veranstaltungen aus Anlaß des Coppernicus-Jahres in Algerien und schließlich den stärkeren Austausch von Journalisten.

62 Siehe PAP in Trybuna Ludu, Nr. 117 vom 27. 4. 1974.

63 Es handelte sich jeweils um eine größere Gruppe polnischer Arzte. Siehe PAP in Zycie Warszawv, Nr. 225 vom 20. 9. 1974.

$64 \mathrm{Im}$ Februar 1973. wurde in Algier der "Plan zur Zusammenarbeit im Gesundheitswesen und in der medizinischen Wissenschaft für 1973 und $1974^{\text {“ }}$ unterzeichnet. Siehe Trybuna Ludu, Nr. 41 vom 11. 2. 1973 . 
tion in der polnischen Hauptstadt - auf Einladung des dortigen Regierungssprechers - mit Mitgliedern der Regierung, in der Abteilung für Propaganda und Pressewesen des Zentralkomitees der Partei, in der Redaktion des Zentralorgans der Partei, in der Hauptverwaltung des Verbandes Polnischer Schriftsteller usw. im Oktober $1973^{65}$ folgte eine erste Vereinbarung über die Zusammenarbeit für das Jahr 1974. Im Februar wurde sie, in bedeutend erweitertem Umfang, in Algier durch ein neues Dokument abgelöst ${ }^{66}$.

\section{Polnische Kulturoffensive}

Die Aktivität Warschauer Kulturschaffender und kultureller Institutionen wurde als letztes Glied in der Kette umfassender Algerienpolitik Polens - mit der Veranstaltung einer Woche der polnischen Kultur, unter Mitwirkung zahlreicher bildender Künstler, Grafiker, Filmschaffender, Musiker, Schriftsteller, Wissenschaftler und Journalisten, im Jahre 1974 eingeleitet. In der Hauptstadt Algier und in weiteren Städten fanden Filme mit nationaler Widerstands- und Unabhängigkeitsthematik - auf beiden Seiten ist die Schicksalsidentität des polnischen und des algerischen Volkes im 19. und im 20. Jahrhundert wiederholt hervorgehoben worden - starkes Interesse in der Öffentlichkeit. Umfang und Intensität der kulturellen Darstellung Polens hatten im Frühjahr 1975 einen solchen Grad erreicht, daß polnischerseits festgestellt wurde, Algerien erlebe eine Zeit der authentischen Offensive polnischer Kultur und polnischer Wissenschaft ${ }^{67}$.

Es entspricht völlig der immer wieder festgestellten und auch weiter zu beobachtenden Konsequenz und Beharrlichkeit in der Verfolgung außenpolitischer, gesellschaftspolitischer und ideologisch-propagandistischer Zielsetzung der Führungskräfte in den Staaten des Ostblocks gegenüber ausgewählten Ländern der Dritten Welt, wenn eingeleitete Kontakte zu sogenannten fortschrittlichen, politischen Gruppierungen oder Bewegungen in diesen Ländern kein einmaliger Vorgang geblieben sind und bleiben, vielmehr zielstrebig ausgebaut und zu verstärkten Aktivitäten, mit dem Merkmal intensiver Propaganda und Indoktrination, geworden sind oder noch werden. Das gilt auch für die polnische Algerienpolitik und findet am Beispiel der Entwicklung der Beziehungen auf parteipolitischer Ebene, somit $z$ wischen den Führungsorganen der PZPR und der Nationalen Befreiungsfront Algeriens, seine Bestätigung.

Dem Besuch der Parteidelegation aus Warschau im November 1973, unter der Leitung des Politbüro-Mitglieds Babiuch ${ }^{68}$, in Algerien - als wichtigstes Ergebnis wurde der beiderseitige Beschluß zu einer sehr engen Zusammenarbeit, auch und vor allem in internationalen, außenpolitischen Fragen, gewertet - folgte schon im Februar 1974 der Gegenbesuch einer Delegation des algerischen Partners, mit dem Mitglied des Revolutionsrats und dem Staatsminister Sherif Belkasem an der Spitze ${ }^{69}$. Wenn der Zweck dieses Gegenbesuchs in der Fortführung der im

\footnotetext{
65 Siehe Trybuna Ludu, Nr. 296 vom 24. 10. 1973.

66 Siehe Trybuna Ludu, Nr. 28 vom 3. 2. 1975

67 Siehe Trybuna Ludu, Nr. 94 vom 24. 4.1975.

68 Babiuch ist für Fragen der inneren Struktur, des Programms und der Satzung der Partei, darüber hinaus noch für die Verbindung $\mathrm{zu}$ den sogenannten fortschrittlichen Bewegungen und befreundeten, brüderlichen, politischen Gruppen und Parteien in anderen Ländern zuständig.

69 Die Einladung hatte Babiuch, im Namen des ZK der PZPR, bereits während seiner Anwesenheit in Algier übermittelt.
} 
November des Jahres davor in der algerischen Hauptstadt begonnenen Gespräche bestand und sich im Willen zur Vertiefung der Beziehungen beider Parteien zueinander, zu noch engerer Zusammenarbeit und darüber hinaus in der Bestätigung der Freundschaft manifestierte, so galt er doch insonderheit der Konsultation in aktuellen Fragen der Außenpolitik. Zu den maßgeblichen Gesprächspartnern der Gäste aus Nordwest-Afrika im Zentralkomitee der Gastgeber zählten denn auch die zuständigen, sachkundigen Funktionäre: Der Sekretär im ZK der PZPR, J. Pinkowski, der Leiter der Außenpolitischen Abteilung im ZK der PZPR, R. Frelek, und dessen Stellvertreter L. Piatkowski ${ }^{70}$.

Nach einem Meinungsaustausch mit dem Mitglied des Politbüros und dem Außenminister in der polnischen Regierung, Stefan Olszowski, zu Schlüsselfragen der internationalen Lage, vor allem im Hinblick auf das Nahost-Problem ${ }^{71}$, bildete der Empfang der Delegation durch den Ersten Sekretär des ZK der PZPR, Edward Gierek, den Höhepunkt und zugleich den Abschluß des Besuchs der algerischen Gäste. Dabei hat der führende polnische Parteifunktionär nicht allein die im November des Vorjahres von Babiuch ausgesprochene Versicherung der Solidarität Polens mit der Politik des Landes der Gäste wiederholt; er brachte auch seine Anerkennung für die gesellschaftspolitischen Veränderungen in jenem Lande, vor allem aber für die hohen Verdienste der Politik Algeriens im Sinne des gesellschaftspolitischen, systemverändernden Fortschritts in der Welt zum Ausdruck ${ }^{72}$.

Die nächste Gelegenheit zu Gesprächen, Erfahrungsaustausch und Konsultation auf bilateraler Parteiebene in der algerischen Hauptstadt eröffneten Ende Oktober 1974 die Veranstaltungen aus Anlaß des 20. Jahrestages der algerischen Revolution. Die von der Regierung der Gastgeber eingeladene Delegation aus Warschau führte indessen nicht allein mit Vertretern der höchsten Organe der Nationalen Befreiungsfront und der Regierung sachdienliche Gespräche. Sie nutzte die Anwesenheit von Abordnungen aus vielen anderen Entwicklungsländern in Algier zu politischen Gesprächen mit Mitgliedern dieser Gruppen. Es bleibt aber noch die nicht nur protokollarische Anwesenheit - etwa als formaler Akt der Höflichkeit - einer Abordnung der polnischen Streitkräfte in Algerien zu verzeichnen. Sie wurde vom stellvertretenden Verteidigungsminister General T. Tuczapski geleitet und reiste mit der Parteidelegation in die polnische Hauptstadt zurück ${ }^{73}$.

\section{Tunesien}

Von vergleichsweise sehr viel geringerem Interesse für Polen, als Handels- und Wirtschaftspartner, aber auch im Hinblick auf engere Beziehungen und Zusammenarbeit in internationalen Fragen und in Fragen der Außenpolitik, ist der dritte und zugleich kleinste Staat in Maghreb, Tunesien, gewesen und offensichtlich geblieben - jedenfalls insoweit das polnisch-tunesische Verhältnis verifizierbar ist. Die Beziehungen beschränken sich im Grunde auf die kulturelle, wissenschaftliche und technische Zusammenarbeit und auf den Warenaustausch. Nach der Unterzeichnung eines Abkommens zur wissenschaftlichen und technischen Zusammenarbeit am 16.9.1961 in Warschau und eines die allgemeine kulturelle Zusammenarbeit

70 Siehe Trybuna Ludu, Nr. 58 vom 7. 2. 1974.

71 Siehe Trybuna Ludu, Nr. 59 vom 28. 2. 1974.

72 Siehe ebenda.

73 Siehe Trybuna Ludu, Nr. 307 vom 3. 11. 1974. 
betreffenden Vertrags am 27. 4. 1966 in der tunesischen Hauptstadt wurde am 6. 5. 1970 in Warschau ein Programm unterzeichnet, das die Vertiefung und Erweiterung mehrerer Formen des kulturellen, wissenschaftlichen und wissenschaftlich-technischen Austauschs in den Jahren 1970 und 1971 zum Inhalt hat ${ }^{74}$. Das nachfolgende, am 27. 4. 1972 wiederum in Tunis vereinbarte Programm erstreckte sich auf die Zusammenarbeit im Hochschulbereich, im Berufsschulwesen, in der Erziehung, im kulturellen und im künstlerischen Bereich im Jahr der Unterzeichnung und im folgenden $\mathrm{Jahr}^{75}$. Die bislang letzten polnisch-tunesischen Gespräche in Warschau führten am 29. 4. $1974 \mathrm{zu}$ einer nochmaligen, vertraglich festgelegten Erweiterung der Zusammenarbeit - u. a. in der Form einer fortgesetzten Beteiligung polnischer Archäologen an den Arbeiten zur Erhaltung der Baudenkmäler in Karthago ${ }^{76}$. Was den Warenaustausch und die wirtschaftliche Zusammenarbeit untereinander anbetrifft, ist jeweils vereinbart worden, die bilaterale Lieferung von Warengütern zu verstärken und die Voraussetzungen zur erweiterten Zusammenarbeit $\mathrm{zu}$ schaffen. Im besonderen wurden die polnischen Ausfuhrkontingente (auf mancherlei Maschinen und Anlagen vor allem für die chemische Industrie und für den Bergbau in Tunesien sowie auf Chemikalien und Schwefel) erhöht und die Listen für polnische Exportgüter nach Tunesien erweitert. Die Wareneinfuhr Polens aus jenem Lande umfaßt neben Zitrusfrüchten, Wein, verschiedenen Konsumgütern vor allem Zinkkonzentrate, Blei, Superphosphat und Futtermittel 7 .

Abschließend sollte nicht unerwähnt bleiben, daß Polen aus dem akuten Mangel an eigenen, qualifizierten Arbeitskräften, Facharbeitern und Spezialisten in den Entwicklungsländern auf seine Weise Nutzen zieht. Mit rund eintausend Fachkräften, die auf Grund von Arbeitsverträgen in Ländern der Dritten Welt, vor allem in Nordafrika und im Nahen Osten, Anfang 1975 tätig gewesen sind ${ }^{78}$, ist auch auf der wirtschaftlichen, wissenschaftlich-technischen, kulturellen Ebene, im Gesundheitswesen u. a. m. die polnische, personelle Präsenz eine bemerkenswerte Realität. 
strategy to exclude the West from the Indian Ocean and its oil transportation routes. But in reality the actual degree of Soviet influence in Somalia in no way approximates control or "satellitization". In fact the political and socio-economic realities of Somalia suggest constraints, difficulties, and responsibilities for the USSR that significantly complicate the realization or extent of Soviet influence in this strategically located country on the Horn of Africa.

\section{The Relations Between Poland and the States of North-West-Africa (Maghreb)}

\section{BY H. L. KRONBERGER}

Following its national interest the communist Poland has established relations to the developing countries and has consequently enlarged them. In doing so they have acted in accordance with the principles of the eastern communist countries, which are proletarian internationalism, establishment of socialist conditions in the world, support of national liberation movements and rebellion, peaceful coexistence and ideological class-struggle. Poland has also established and developed the relations to the States of North-West-Africa.

Morocco and Poland could practically continue the relations they had during the two world wars. Economic interests, positive experiences in the building of complete branches of industry on the Polish side, high specialization in the Polish industry of capital goods and last but not least the question of tradition had in 1974 led to the signing of a trade agreement and of several supply contracts for the Moroccan railway. At the beginning of 1975 out of all eastern communist states Poland took the lead in the field of foreign trade and economic co-operation with Morocco. In the politico-ideological field the Polish government aims at changing the internal structure and the system of the kingdom of the country and at supporting the powers of socialism.

Poland could establish relations to Algeria only after it had received its independence in 1962. The economic relations between both countries have been fairly moderate since then but after the visit of a Polish party delegation in Algeria end of 1973 they received a strong stimulation. For the Polish government it was, however, more important to deepen the ideological and socio-political co-operation with the Algerian Liberation Front and the common battle against the so-called imperialism and neo-colonialism. The Polish effort to establish a communist system in Algeria was in so far successful as the Algerian government gave the assurance that the Polish credits for investment should contribute to the changing of the economic structures in the country according to the socialist model. Apart from Warsaw's efforts to increase its connection to the National Liberation Front of Algeria it is willing to establish contacts to communist and revolutionary powers all over the world and to make propaganda among the Algerian population for a closer relation to Poland and the other eastern communist countries. The variety and intensity of the Algerian-Polish relationship and their strong co-operation in the field of economy, technical science, culture and journalism should not hide the fact that the Polish policy towards Algeria is mainly characterized by political propaganda and ideological indoctrination. 
The Polish relations towards Tunesia are more or less confined to a cultural, scientific and technical co-operation and to the exchange of goods. In 1974 an enlargment of the relations has been stipulated by contract.

\section{Theories of Imperialism and Modernization}

\section{By Kurt-Peter SchütT}

Theories of imperialism and modernization are the most important approaches to the analysis of developing societies. Both of these conflicting theories lay exclusive claims to an adequate access to the problem of underdevelopment; each is producing extremly different results. Right here is raised the problem of how to qualify their respective theoretical capacities. This article elaborates as well on the practical considerations underlying both concepts prior to theory formation as on the theoretical assumptions and implications and their combined explanatory impact. First of all, the theory of imperialism as developed by W. I. Lenin is briefly overviewed and rejected as empirically invalid. After this follows a discussion of the neo-marxist theory of imperialism which is rooted in the classical theory and was first formulated by P. A. Baran. This revisionist concept forms the basis of the "theory of dependence" and "theories of periphery capitalism" which have come to dominate the current scientific debate about developing nations. The final consideration is an evaluation of Baran's theory in view of the theory of modernization that is basically influenced by functionalist thinking. The results point to onesidedness of both concepts with regard to the premises, objectives and subjects of research. The neo-marxist theory of imperialism reduces problems of development and underdevelopment to narrow economic causes by applying a strict politico-economic interpretation. The modernization theory is rather a theory of action which neglects conflicts of authority and interest resulting from socioeconomic conditions. Any future research on developing countries in the social sciences should proceed on the premise of combining politico-economic analysis and theories of action in order to achieve a really informative theory of development and underdevelopment capable of better explanations. 